\title{
Impact of different therapies on the survival of patients with stage I-IIA cervical cancer with intermediate risk factors
}

\author{
Jichan Nie", Qinjiao Wu", Anqi Yan, Zhiyong Wu \\ Gynecology Department, Shanghai Obstetrics and Gynecology Hospital of Fudan University, Shanghai, China \\ Contributions: (I) Conception and design: J Nie, Z Wu; (II) Administrative support: Z Wu; (III) Provision of study materials or patients: Q Wu, A Yan; \\ (IV) Collection and assembly of data: All authors; (V) Data analysis and interpretation: J Nie, Q Wu; (VI) Manuscript writing: All authors; (VII) Final \\ approval of manuscript: All authors. \\ "These authors contributed equally to this work. \\ Correspondence to: Zhiyong Wu. Gynecology Department, Shanghai Obstetrics and Gynecology Hospital of Fudan University, No. 419 Fangxie Road, \\ Shanghai 200011, China. Email: wuzhiyong@fudan.edu.cn.
}

\begin{abstract}
Background: The aim of this study was to determine the effects of different therapies on patients with cervical cancer (CC) with intermediate risk factors.

Methods: Clinicopathological data of 596 patients diagnosed with stage I-IIA CC at the Obstetrics and Gynecology Hospital of Fudan University between January 2013 and November 2015 were retrospectively reviewed. Of the patients, 500 patients received adjuvant therapy including chemotherapy (CT), radiotherapy (RT), and sequential chemotherapy and radiotherapy $(C T+R T)$. Patients who displayed at least one intermediate risk factor number were screened.

Results: The median follow-up was 62 months. The 5 -year progression-free survival (PFS) and overall survival (OS) of the entire cohort were $90.4 \%$ and $90.9 \%$, respectively. Univariate analysis showed that tumor stage, tumor size, pathological type, lymphovascular space invasion, and numbers of medium risk factors were not risk factors for early-stage CC. Compared with the control group, patients who received CT, RT, or CT + RT showed improved PFS and OS $(\mathrm{P}<0.05)$. The RT group had lower PFS and OS than the CT and CT + RT groups $(\mathrm{P}<0.05)$. Among the 318 patients with a single intermediate risk factor, 297 patients received CT, RT, and CT + RT benefit from adjuvant therapy $(\mathrm{P}<0.05)$. Of the 253 patients with high-risk factors, 220 patients received CT, RT and CT + RT get improved PFS and OS $(\mathrm{P}<0.05)$.

Conclusions: Patients who received adjuvant therapy had better postoperative outcomes than those who did not receive adjuvant therapy. Patients had CT alone or CT combined with RT had better efficacy than those had RT alone.
\end{abstract}

Keywords: Cervical cancer (CC); intermediate risk factors; progression-free survival (PFS); overall survival (OS)

Submitted Nov 11, 2020. Accepted for publication Dec 29, 2020.

doi: $10.21037 / \mathrm{atm}-20-7679$

View this article at: http://dx.doi.org/10.21037/atm-20-7679

\section{Introduction}

Cervical cancer (CC) imposes a heavy burden on women worldwide, with 529000 new diagnoses annually (1). Despite the progress that has been made and the increased awareness of human papillomavirus over the last decade, $10-20 \%$ of CC patients go on to develop recurrence and metastasis after surgery, among which the lung is the most common site of metastasis (2). The recurrent CC remains challenging, especially in less developed regions. Currently, treatment options for recurrent CC include surgery, radiotherapy (RT), chemoradiotherapy, and chemotherapy (CT), depending on the stage and response of the tumor $(3,4)$. Surgery or RT is recommended for patients with stage I-IIA disease. Surgery has the advantages of improving survival and the quality of life of patients. Intermediate risk factors for CC include lymphovascular space invasion, 
depth of stromal invasion, and tumor size. The treatment guidance and options of patients with intermediate risk factors for CC remain unclear. Here, we conducted a longterm investigation into the survival rate of women with CC, assessed the possible independent risk factors for CC, and evaluated the optimal therapeutic method for CC patients with one or more intermediate risk factors.

We present the following article in accordance with the STROBE reporting checklist (available at http://dx.doi. org/10.21037/atm-20-7679).

\section{Methods}

\section{Study population}

A total of 596 patients with a diagnosis of I-IIA CC in accordance with the International Federation of Gynecology and Obstetrics (FIGO) staging system (2018) were included in this study. The inclusion criteria for patients were: received radical hysterectomy and pelvic lymph node dissection; complete clinical data; and postoperative pathology showing one or more intermediate risk factors (LVSI+, DSI+, TS $>4 \mathrm{~cm}$ ). The exclusion criteria were: a history of malignancy or high-risk factors (positive nodes, positive margins, or positive parametria). A representative sample of the pathology slides, operative notes, and planning films for RT were reviewed for each patient. All procedures performed in this study involving human participants were in accordance with the Declaration of Helsinki (as revised in 2013). Individual consent for this retrospective analysis was waived. The study protocol was approved by the ethics committee of Obstetrics and Gynecology Hospital of Fudan University (No. 2020-203).

\section{Regimen for postoperative adjuvant therapy}

Most patients with intermediate risk factors received CT, RT, or sequential CT and RT. The control group comprised patients who refused recommended postoperative complementary treatment and requested observation. Patients in the CT group received platinumbased combination CT every 3 weeks for four cycles with cisplatin/lobaplatin/carboplatin $($ AUC $=5)+$ paclitaxel $\left(135 \mathrm{mg} / \mathrm{m}^{2}\right) /$ docetaxel/paclitaxel liposomes. In the RT group, RT alone was administered approximately 4 weeks after the operation. The RT target area included the preoperative tumor site and the drainage area of the pelvic lymph nodes. A total dose of 45-50 Gy of RT was delivered in 25 fractions (in fractions of 1.8-2.0 Gy,
Monday to Friday), using three-dimensional conformal RT or intensity-modulated RT. In the CT + RT group, CT was performed every 3 weeks for four cycles, with the first one or two cycles given before RT and the remaining cycles conducted after the completion of RT.

\section{Follow-up}

After treatment, all patients were followed up every 3 months for the first 2 years and every 6 months for the next 3 years. The follow-up methods included phone calls, questionnaires, and outpatient visits. Recurrence was diagnosed based on the results of computed tomography (CT) or positron emission tomography-CT (PET-CT). Overall survival (OS) was calculated from the time of the initial diagnosis of CC until the date of cancer-related death. Progression-Free-Survival was defined as the time from random to the first occurrence of disease progression or death of any cause.

\section{Statistical analysis}

Data analysis was performed using SPSS 17.0 (IBM Corp., New York, USA). Differences in the proportion were measured using the $\chi^{2}$ test. For continuous data, Student's $t$-test was performed. Logistic regression models were used for univariate analysis. Survival curves were established using the Kaplan-Meier method. Statistical significance was set at $\mathrm{P}<0.05$.

\section{Results}

\section{Patient baseline characteristics}

The follow-up rate was $95.8 \%$, with 25 of the patients lost follow-up. The clinical characteristics of the 571 patients that met the inclusion criteria are shown in Table 1, including age, tumor stage, pathological type, tumor size, stromal invasion, lymphovascular space invasion, and postoperative complementary therapy. Of the 571 patients included, 318 cases had 1 intermediate risk factor and 253 cases had $\geq 2$ intermediate risk factors. The number of intermediate risk factors is shown in Figure 1.

\section{Survival analysis of CC patients}

The survival curves of patients who received different adjuvant therapies are shown in Figure 2. The 5-year 
Table 1 Clinical data of cervical cancer cases

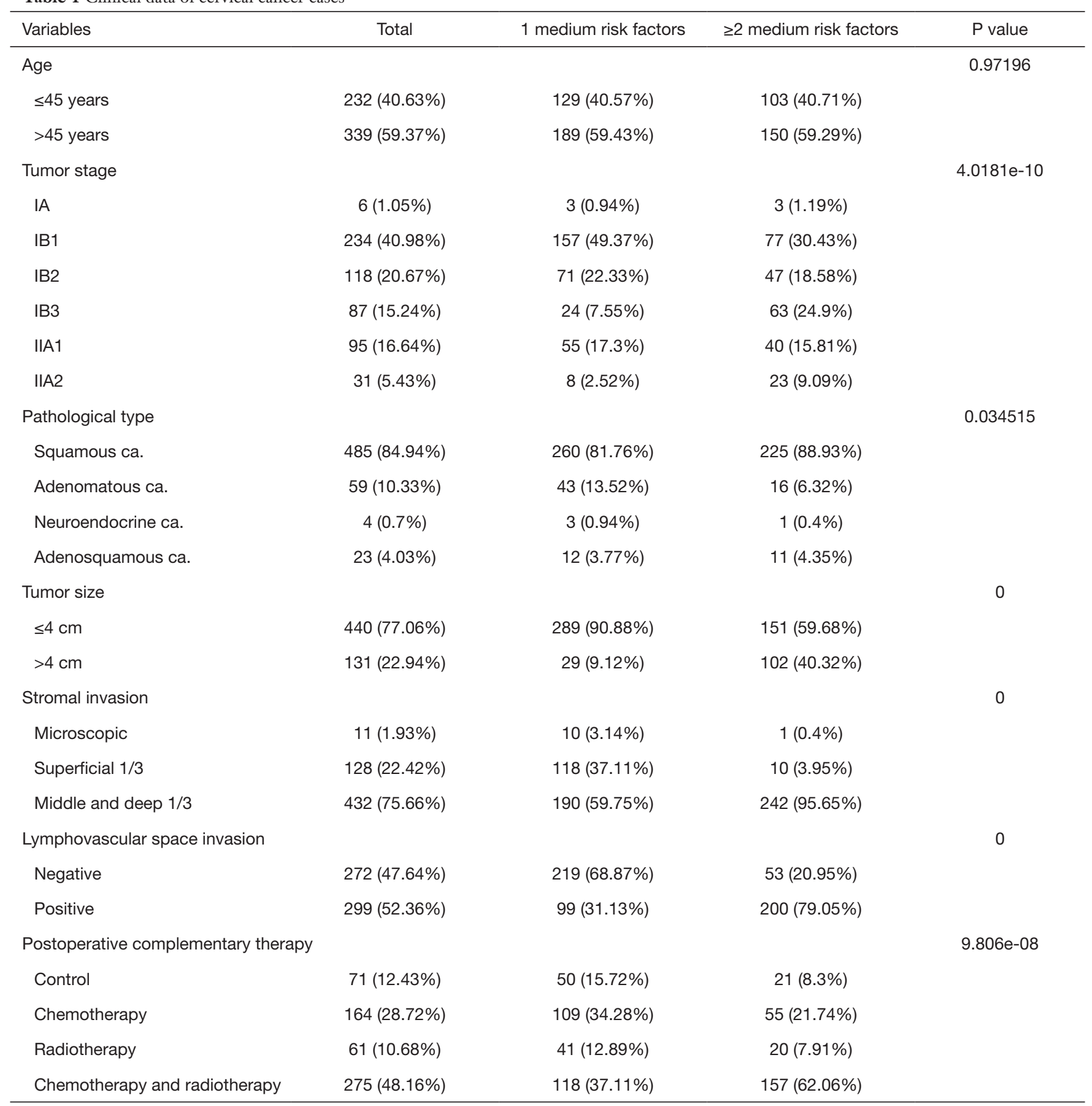

progression-free survival (PFS) rate for the entire cohort was $90.4 \%$ (Figure $2 A$ ). The 5 -year PFS rate for the control group was $74.6 \%$, and rates of $93.9 \%, 93.4 \%$, and $91.6 \%$ for CT, RT, and CT + RT groups, respectively (Figure 2B). The OS rate for all patients was $90.9 \%$ (Figure $2 A$ ). The OS rate for the control group was $76.0 \%$, and rates of $93.9 \%$, 93.4\%, and $92.4 \%$ for the CT, RT, and CT + RT groups, respectively (Figure 2C).

In terms of the number of intermediate risk factors, the PFS rates of low-risk patients with a single intermediate 


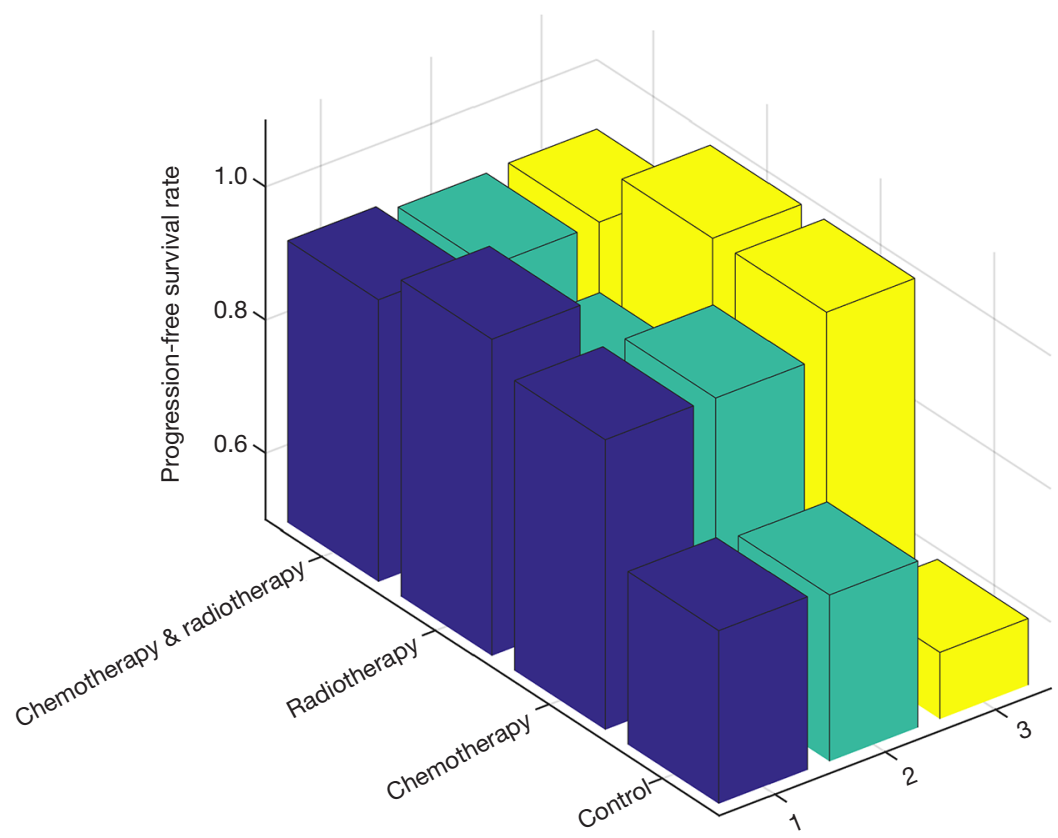

Counts of medium risk factors

Figure 1 Bar chart showing the numbers of intermediate risk factors for the patients in each treatment group.

risk factor in the control, CT, RT, and CT + RT groups were $76.0 \%, 93.6 \%, 97.6 \%$, and $92.4 \%$, respectively (Figure 2D). The OS rates of the control, CT, RT, and CT + RT groups were $78.0 \%, 93.6 \%, 97.6 \%$, and $93.2 \%$, respectively (Figure 2E). For high-risk patients with $\geq 2$ intermediate risk factors, the PFS rates of the control, CT, RT, and CT + RT groups were 71.4\%, 94.5\%, 85\%, and $91.0 \%$, respectively (Figure $2 F$ ). The OS rates of the control, CT, RT, and CT + RT groups were $71.4 \%$, $94.5 \%, 85.0 \%$, and $91.7 \%$, respectively (Figure $2 G$ ). The $\mathrm{PFS}$ and OS rates in the RT group were significantly lower than those in the CT and CT + RT groups $(\mathrm{P}<0.05)$. These results indicated that patients who received adjuvant therapy had longer PFS and OS than those who did not receive adjuvant therapy, regardless of the number of intermediate risk factors $(\mathrm{P}<0.05)$. No significant difference was found in the PFS or OS rate between the various adjuvant therapies $(\mathrm{P}>0.05)$. The recurrence rates of the control, CT, RT, and CT + RT groups are shown in Table 2. Patients in the control group had a higher rate of recurrence than those in the CT, RT, and CT + RT groups $(\mathrm{P}>0.05)$. Patients who received $\mathrm{RT}$ had a higher rate of recurrence than those in the $\mathrm{CT}$ and $\mathrm{CT}+\mathrm{RT}$ groups $(\mathrm{P}<0.05$; Figure 3).

\section{Prognostic factors for $C C$}

Univariate analysis was performed to identify the possible prognostic factors. Tumor stage, tumor size, pathological type, lymphovascular space invasion, and numbers of intermediate risk factors were not found to be independent predictors of poor OS in early-stage CC ( $\mathrm{P}>0.05$; Table 2).

\section{Discussion}

Methods of adjuvant therapy have become a hotspot of CC management. The present study aimed to determine the survival benefit of adjuvant therapy in patients with earlystage CC. As reported, early-stage invasive disease accounts for $50-75 \%$ of all CC cases in the United States (5). Based on the FIGO system, early-stage CC refers to stages IAIIA, and some oncologists consider stages IB3 and IIA2 to be advanced disease $(6,7)$. Our data showed that early-stage CC patients who received postoperative adjuvant therapy had a relatively favorable prognosis compared those who didn't receive adjuvant therapy.

It is unclear whether the number of intermediate risk factors is an indication for adjuvant therapy, and different guidelines are inconsistent. In the AGO guidelines, for instance, RT or CT is recommended for patients with 
A

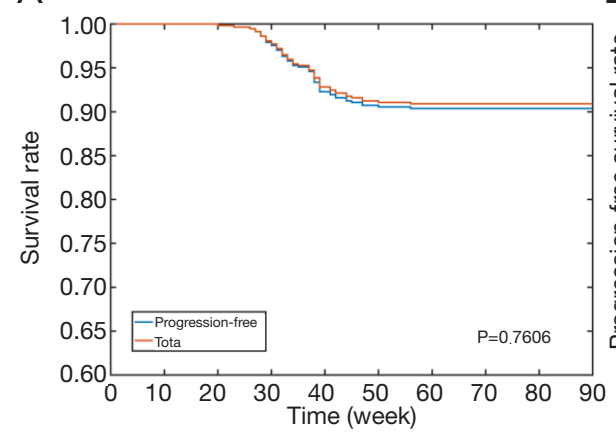

D

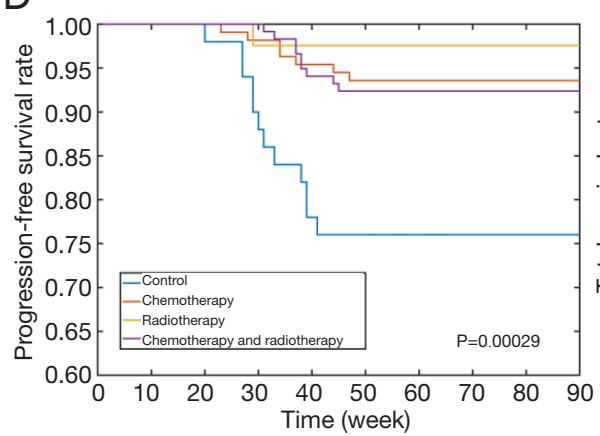

G

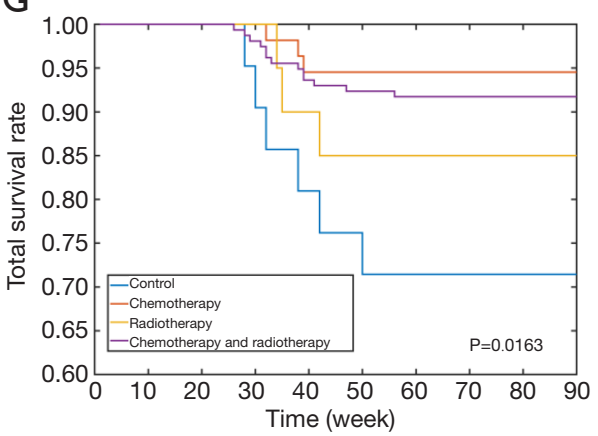

B

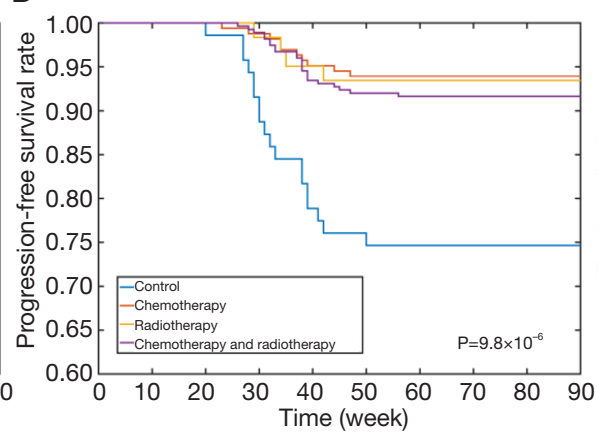

$E_{1}$

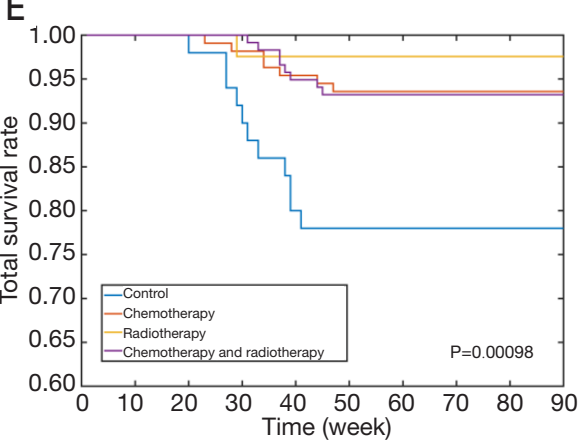

C

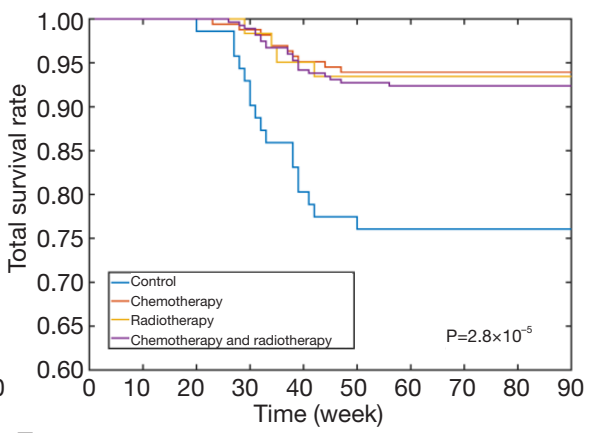

$\mathrm{F}$

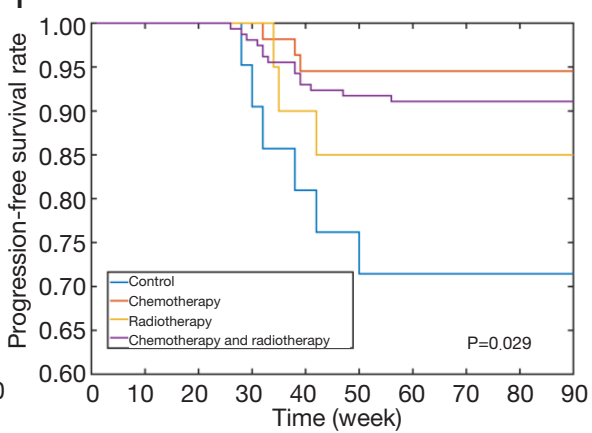

Figure 2 Comparison of different adjuvant therapy regimens [chemotherapy (CT), radiotherapy (RT), and sequential chemotherapy and radiotherapy $(\mathrm{CT}+\mathrm{RT})]$. (A) Survival curve of the entire cohort; (B) Progression-free survival (PFS) of patients treated with various adjuvant therapies; (C) Overall survival (OS) of patients treated with various adjuvant therapies; (D) PFS of patients with a single intermediate risk factor; (E) OS of patients with a single intermediate risk factor; (F) PFS of patients with $\geq 2$ intermediate risk factors; (G) OS of patients with $\geq 2$ intermediate risk factors.

a single intermediate risk factor. Takeshima et al. (8) reported that after treatment with adjuvant CT alone, the rate of recurrence in patients with intermediate was $3.3 \%$ and the rate of recurrence in patients with high risk factors was $8.6 \%$. In this study, the patients did not receive preoperative neoadjuvant CT due to their early disease stage. According to our data, patients with $\geq 1$ intermediate risk factor in the CT, RT, and CT + RT groups had increased survival rates compared to those in the control group. Notably, in the present study, the recurrence rate in patients with $\geq 2$ intermediate risk factors who received RT was $15.8 \%$, which is higher than that reported in a previous study (9). Furthermore, the PFS and OS in the RT group were significantly lower than those in the other groups. The influence of tumor stage on RT and even the guidelines for RT are still controversial. A meta-analysis revealed that concurrent $\mathrm{RT}$ and $\mathrm{CT}$ was effective than RT alone (10), which is consistent with the findings of the present study. It has been reported that relapse in patients treated with RT was on account of a higher risk of 
Table 2 Univariate analysis of progression-free survival and clinical-pathological characteristics

\begin{tabular}{|c|c|c|c|c|}
\hline Variables & $\mathrm{N}$ & Recurrence/death & $\chi^{2}$ & $P$ value \\
\hline IA & 6 & $0(0 \%)$ & & \\
\hline IB1 & 234 & $23(9.83 \%)$ & & \\
\hline IB2 & 118 & $10(8.47 \%)$ & & \\
\hline IIA1 & 95 & $8(8.42 \%)$ & & \\
\hline IIA2 & 31 & $2(6.45 \%)$ & & \\
\hline Tumor size & & & 1.3016 & 0.25393 \\
\hline$\leq 4 \mathrm{~cm}$ & 440 & $39(8.86 \%)$ & & \\
\hline Squamous ca. & 485 & $48(9.9 \%)$ & & \\
\hline Adenomatous ca. & 59 & $7(11.86 \%)$ & & \\
\hline Neuroendocrine ca. & 4 & $0(0 \%)$ & & \\
\hline Adenosquamous ca. & 23 & $0(0 \%)$ & & \\
\hline Lymphovascular space invasion & & & 0.26144 & 0.60913 \\
\hline Negative & 272 & $28(10.29 \%)$ & & \\
\hline Positive & 299 & $27(9.03 \%)$ & & \\
\hline Counts of intermediate-risk factors & & & 0.21676 & 0.64152 \\
\hline
\end{tabular}

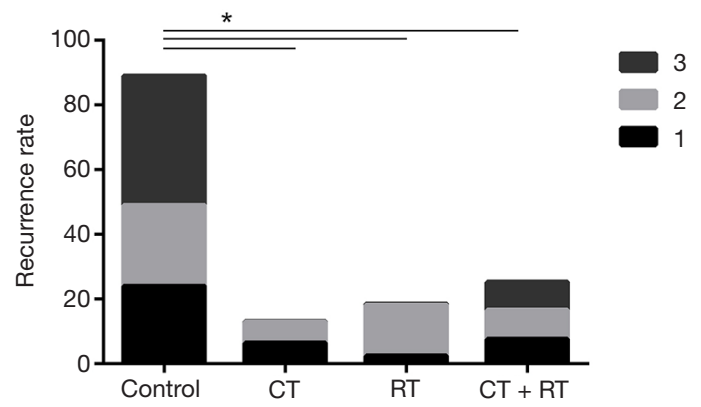

Figure 3 Recurrence rate of patients with different numbers of intermediate risk factors. Recurrence rates of patients had no adjuvant therapy or different adjuvant therapy regimens [chemotherapy (CT), radiotherapy (RT), and sequential chemotherapy and radiotherapy $(\mathrm{CT}+\mathrm{RT})]$ were calculated. ${ }^{*} \mathrm{P}<0.05$. complications (11), which may explain the higher recurrence rate in this study. Data on the use of CT in patients with stage IA-IIA CC is sufficient, including clinical outcome and follow-up research. A group study showed that the addition of paclitaxel to cisplatin, which was also applied in this study, contributed to rises in the response rate, PFS, and OS (12). Apparent evidence of the advantage of adjuvant therapy has been illustrated in other studies, as eradicates residual and microscopic disease (13-17). Generally, patients treated with postoperative adjuvant therapy who have $\geq 2$ intermediate risk factors display a similar curative effect as those with a single intermediate risk factor.

Previous studies have identified stage, the number of pelvic lymph nodes, and histology as a strong prognosis factor of CC (18-23). The effects of pathological type, 
lymphovascular space invasion, and numbers of intermediate risk factors on PFS and OS have not been determined. In the present study, tumor stage, tumor size, pathological type, lymphovascular space invasion, and numbers of intermediate risk factors were not found to be prognostic factors for early-stage CC patients who received CT, RT, or $\mathrm{CT}+\mathrm{RT}$ as an adjuvant therapy.

In conclusion, we found that adjuvant therapy, especially $\mathrm{CT}$ and $\mathrm{CT}+\mathrm{RT}$, contributed to increased survival in patients with early-stage CC. This was a relatively large, population-based study of adjuvant therapy with a longterm follow-up. Based on the retrospective data analyzed in this study, CT or CT plus RT is recommended for patients with early-stage CC.

\section{Acknowledgments}

Funding: This research was funded by the Shanghai Health System Talent Training Program (grant no. 2018YQ60).

\section{Footnote}

Reporting Checklist: The authors have completed the STROBE reporting checklist. Available at http://dx.doi. org/10.21037/atm-20-7679

Data Sharing Statement: Available at http://dx.doi. org/10.21037/atm-20-7679

Conflicts of Interest: All authors have completed the ICMJE uniform disclosure form (available at http://dx.doi. org/10.21037/atm-20-7679). The authors have no conflicts of interest to declare.

Ethical Statement: The authors are accountable for all aspects of the work in ensuring that questions related to the accuracy or integrity of any part of the work are appropriately investigated and resolved. All procedures performed in this study involving human participants were in accordance with the Declaration of Helsinki (as revised in 2013). Individual consent for this retrospective analysis was waived. The study protocol was approved by the ethics committee of Shanghai Obstetrics and Gynecology Hospital of Fudan University (No. 2020-203).

Open Access Statement: This is an Open Access article distributed in accordance with the Creative Commons Attribution-NonCommercial-NoDerivs 4.0 International
License (CC BY-NC-ND 4.0), which permits the noncommercial replication and distribution of the article with the strict proviso that no changes or edits are made and the original work is properly cited (including links to both the formal publication through the relevant DOI and the license). See: https://creativecommons.org/licenses/by-nc-nd/4.0/.

\section{References}

1. Ferlay J, Shin HR, Bray F, et al. Estimates of worldwide burden of cancer in 2008: GLOBOCAN 2008. Int J Cancer 2010;127:2893-917.

2. Ki EY, Lee KH, Park JS. A Clinic opathological Review of Pulmonary Metastasis from Uterine Cervical Cancer. Cancer Res Treat 2016;48:266-72.

3. Yee GP, de Souza P. Current and potential treatments for cervical cancer. Curr Cancer Drug Targets 2013;13:205-20.

4. Shu T, Zhao D, Li B, et al. Prognostic evaluation of postoperative adjuvant therapy for operable cervical cancer: 10 years' experience of National Cancer Center in China. Chin J Cancer Res 2017;29:510-20.

5. Khanna N, Rauh LA, Lachiewicz MP. Margins for cervical and vulvar cancer. J Surg Oncol 2016;113:304-9.

6. Somashekhar SP. Management of Early Stage Cervical Cancer. Rev Recent Clin Trials 2015;10:302-8.

7. Pecorelli S. Revised FIGO staging for carcinoma of the vulva, cervix, and endometrium. Int J Gynaecol Obstet 2009;105:103-4.

8. Takeshima N, Umayahara K, Fujiwara K.et al. Treatment results of adjuvant chemotherapy after radical hysterectomy for intermediate- and high-risk stage IB-IIA cervical cancer. Gynecol Oncol 2006;103:618-22.

9. Sun HY, Tang Q, Chen JH, et al. Cisplatin concurrent chemoradiotherapy vs adjuvant radiation in stage IB/IIA cervical cancer with intermediate risk factors, treated with radical surgery: a retrospective study. Onco Targets Ther 2018;11:1149-55.

10. Vordermark D. Radiotherapy of Cervical Cancer. Oncol Res Treat 2016;39:516-20.

11. Rogers L, Siu SS, Luesley D, et al. Radiotherapy and chemoradiation after surgery for early cervical cancer. Cochrane Database Syst Rev 2012;(5):CD007583.

12. Moore DH, Blessing JA, McQuellon RP, et al. Phase III study of cisplatin with or without paclitaxel in stage IVB, recurrent, or persistent squamous cell carcinoma of the cervix: a Gynecologic Oncology Group Study. J Clin Oncol 2004;22:3113-9. 
13. Ilancheran A. Neoadjuvant chemotherapy in cervical cancer in pregnancy. Best Pract Res Clin Obstet Gynaecol 2016;33:102-7.

14. Kumar L. Integrating Chemotherapy in the Management of Cervical Cancer: A Critical Appraisal. Oncology 2016;91 Suppl 1:8-17.

15. Falcetta FS, Medeiros LR, Edelweiss MI, et al. Adjuvant platinum-based chemotherapy for early stage cervical cancer. Cochrane Database Syst Rev 2016;11:CD005342.

16. Kumar L, Harish P, Malik PS. Chemotherapy and targeted therapy in the management of cervical cancer. Curr Probl Cancer 2018;42:120-8.

17. Phillips P. Hysterectomy With Radiotherapy or Chemotherapy or Both for Women With Locally Advanced Cervical Cancer. Clin Nurse Spec 2017;31:189-90.

18. Pieterse QD, Kenter GG, Gaarenstroom KN, et al. The number of pelvic lymph nodes in the quality control and prognosis of radical hysterectomy for the treatment of cervical cancer. Eur J Surg Oncol 2007;33:216-21.

19. Kim HS, Kim JH, Chung HH, et al. Significance of numbers of metastatic and removed lymph nodes in FIGO

Cite this article as: Nie J, Wu Q, Yan A, Wu Z. Impact of different therapies on the survival of patients with stage I-IIA cervical cancer with intermediate risk factors. Ann Transl Med 2021;9(2):142. doi: 10.21037/atm-20-7679 stage IB1 to IIA cervical cancer: Primary surgical treatment versus neoadjuvant chemotherapy before surgery. Gynecol Oncol 2011;121:551-7.

20. Park KJ, Braschi-Amirfarzan M, DiPiro PJ, et al. Multimodality imaging of locally recurrent and metastatic cervical cancer: emphasis on histology, prognosis, and management. Abdom Radiol (NY) 2016;41:2496-508.

21. Kang YJ, O'Connell DL, Lotocki R, et al. Effect of changes in treatment practice on survival for cervical cancer: results from a population-based study in Manitoba, Canada. BMC Cancer 2015;15:642.

22. Galic V, Herzog TJ, Lewin SN, et al. Prognostic significance of adenocarcinoma histology in women with cervical cancer. Gynecol Oncol 2012;125:287-91.

23. Lee JY, Kim YT, Kim S, et al. Prognosis of Cervical Cancer in the Era of Concurrent Chemoradiation from National Database in Korea: A Comparison between Squamous Cell Carcinoma and Adenocarcinoma. PLoS One 2015;10:e144887.

(English Language Editor: J. Reynolds) 